Pub. Mat. UAB

vol. 28 No 2-3 Set: 1984

\title{
BLOW UP ON ENERGY LEVELS IN CELESTIAL MECHANICS
}

\author{
Ernesto A. Lacomba *
}

We want to describe here how to study the asymptotic behavior of total collapse and escape orbits for the $n$ rody problem of celestial mechanics by using blow up procedures..This approach was begun for total collapse motion by Mc Gehee 19| in 1974, and has been subseguently exploited by many authors (see Devaney $|2|$, and references therein).

The application of said procedures for escape motion by Lacomba and Simb $|7|$ appeared in 1982, and it has been refined since then, by this author $|3|,|5|,|6|$. We will also refer in the examples to the expository paper $|4|$.

Except for negative energy escape behavior, we can give a general method for describing the blow up, and describe asymptotic motions with a little more effort. However, only when the number of degrees of freedom is small it is possible to have a more or less global picture.

* This research was partially supported by CONACYT (Mexico) grant PCCBNAL 790178. 
In general an n-body problem can be stated in the

form

$$
\begin{aligned}
& \dot{q}=\mathrm{pA}^{-1} \\
& \dot{\mathrm{p}}=\operatorname{grad} \mathrm{v}(\mathrm{q})
\end{aligned}
$$

where $\mathrm{P} \varepsilon \mathbb{R}^{\mathrm{k}}, \mathrm{q} \in \mathrm{N} \subset \mathbb{R}^{\mathrm{k}}$ the set $\mathrm{N}$ is an open cone, and $\mathrm{U}: \mathrm{N}+\mathbb{R}^{+}$(potential energy) is analytic homogeneous of degree-1. The kxk matrix $A$ is positive definite. The Hamiltonian or total energy is $\mathrm{H}(\mathrm{q}, \mathrm{p})=\mathrm{K}(\mathrm{p})-\mathrm{U}(\mathrm{q})$ where

$$
K(p)=1 / 2 \mathrm{pA}^{-1} \mathrm{p}^{\mathrm{t}}
$$

is the kinetic energy. Given $h \in \mathbb{R}$, we define the corresponding energy level by $E_{h}=\left((q, p) E N \times \mathbb{R}^{k}: H(q, p)=h\right\}$. The equation defining it or energy relation is witten as

$$
\text { (2) } K(p)=U(q)+h \text {. }
$$

The projection of $\mathrm{E}_{\mathrm{h}}$ to configuration space is the so called Hill region $u+h \geq 0$. This is a manifold with boundary $\mathrm{U}=-\mathrm{h}$ (zero velocity points).

If $h<0$, in some sense $U$ as attracting potential predominates over $K$, energy due to the motion (see (2)), giving the possibility of bounded or recurrent motions.

If on the contrary $h>0$, then $k$ predominates over U, making it easier to escape. 
Eor example in the n-body problem in $\mathbb{R}^{d}$ ( $d=1,2$ or 3 dimensions) with the usual coordinates we take $q=\left(x_{1}, \ldots, x_{n}\right)$, $p=\left(m_{1} \dot{x}_{1}, \ldots, m_{n} \dot{x}_{n}\right)$ where $x_{i}, \dot{x}_{i} \in \mathbb{R}^{d}$ are positions and velocities of the bodies respectively. Then

$$
u\left(x_{1}, \ldots, x_{n}\right)=\sum_{i<j} G \frac{m_{i} m_{j}}{r_{j i}}
$$

where $r_{j i}=\left|x_{j}-x_{i}\right|$ and $G$ is the gravitational constant. The kinetic energy is simply

$$
K(p)=\frac{1}{2} \sum_{i} m_{i}\left|\dot{x}_{i}\right|^{2}
$$

We remark that for an n-body problem system (1) has no equilibrium points, since forces are internal and attracting. on the other hand, (1) does have singularities, corresponding to collisions of 2 or more bodies. It is an open question if more complicated singularities can occur for $n>3$ or a $>1$. Of these singularities, binary collisions can be treated by the so called regularization $|8|$, while total collapse for any number of bodies is studied by a blow up.

1. Blow up at the origin $|9|$. The idea is to pass to "polar coordinates" in configuration $q$-space with a corresponding consistent change in momenta variables so that the energy relation is not singular at $q=0$ any more. Since energy relation corresponds to a fixed energy $h$, we work on a fixed energy level. Since differential equations will still stay singular, we take an additional change of time scale. 
The natural radial coordinate or "norm" is the radius of inertia $r=\sqrt{\mathrm{gAq}^{t}}$, with respect to which unit vectors or "angular" variables are $Q=r^{-1} q$. Substitution into (2) by taking into account the homogeneity of $U$ gives

$$
r K(p)=U(Q)+r h
$$

Since $K$ is also homogeneous but of degree 2 \{quadratic), the right momenta transformation is $\mathrm{p}=\mathrm{r}^{1 / 2} \mathrm{p}$, giving the energy relation

$$
\text { (3) } \mathrm{K}(\mathrm{P})=\mathrm{U}(\mathrm{Q})+r \mathrm{~h} \text {, }
$$

already regular at $r=0$ (i.e.q $=0)$.

When equations (1) are written in terms of the new coordinates $(r, 0, P)$ they are stili singular at $r=0$. This singularity is easily removed through the change of time scale $d t=r^{3 / 2} d \tau$, which slows down orbits where $r \rightarrow 0$. Approach to total collapse of the system $I=0$ takes place in infinite T-time (asymptoticaliy), while it happens in finite physical t-time. We get the system

$$
\text { (4) } \begin{aligned}
r^{\prime} & =\mathrm{rV} \\
\mathrm{Q}^{\prime} & =\mathrm{PA}^{-1}-\mathrm{VQ} \\
\mathrm{P}^{\prime} & =\operatorname{grad} \mathrm{U}(Q)+(\mathrm{V} / 2) \mathrm{P},
\end{aligned}
$$

where $"=\mathrm{d} / \mathrm{d} \tau, \mathrm{v}=\mathrm{Q} \cdot \mathrm{P}, \mathrm{r}>0$ and coordinate $Q$ takes values in the submanifold.

$$
\text { (5) } S=\left\{Q E N ; \quad Q^{A} Q^{t}=1\right\}
$$


The new momenta $P$ is constrained by the energy relation (3). We verify from (4) that the vector field is still defined for $r=0$. In the new coordinates the flow has been extended to the boundary $C$ (obtained by setting $r=0$ ) of the following manifold with boundary

$$
E_{h} \cup C=\{(r, Q, P): r \geq 0, Q E S, K(P)=U(Q)+r h\}
$$

Since $r=0$ implies $r^{*}=0$ in (4), C is an invariant manifold for the flow. Orbits in $E_{h}$ going to or coming from total coliapse will now approach $C$ asymptotically as $\tau \rightarrow \pm \infty$.

In terms of the physical problem, $C$ and its flow are fictitious but give information about total collapse.

There are equilibrium points for the vector field (4). They belong to $C(r=0)$ as expected, since we remarked above that (I) has no equilibrium points.

Said points are defined by the equations

$$
\text { (4) } \begin{aligned}
& \text { grad } U(Q)=-U(Q) Q A \\
& P_{ \pm}= \pm \sqrt{2 U Q A}
\end{aligned}
$$

The solutions to the first equation are known as central configurations of the system and they are interpreted as the critical points of the restriction $U / s$. We state the most important result $|1|$ for the study of the flow: 
Proposition 1.- If $\mathrm{U} / \mathrm{s}$ is a Morse function, then all the (isolated) equilibrium points for the flow on $c$ are hyperbolic and the flow is gradient-like with respect to the Eunction $v$.

We will end this section by describing a couple of examples (see $|4|$, where figures are shown for each transforma tion)

Example 1. Consider the kepler problem in 1 dimen sion. In this case the differential equations are

$$
\begin{aligned}
& \dot{x}=y \\
& \dot{y}=-x^{-2}
\end{aligned}
$$

for $x>0, y \in \mathbb{R}$. The energy relation reads as follows, giving solutions as level. curves (see figure 1, right).

$$
\text { (6) } y^{2} / 2=x^{-1}+h \text {. }
$$

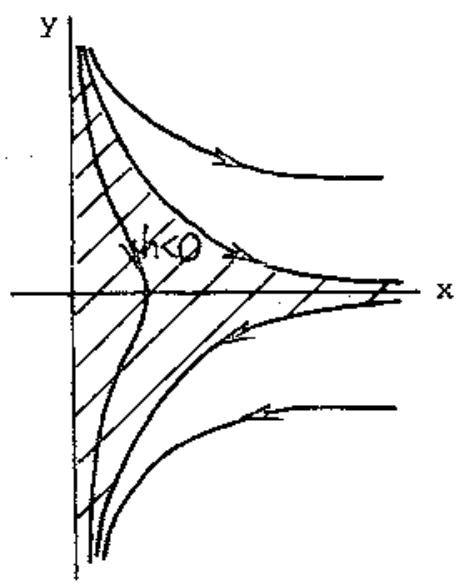

Notice that $y \rightarrow \pm \infty$ if $x+0$

Fig. I

Blow up at origin $x=0$ for one degree of freedom amounts to keep' $x$ as radial coordinate, with a momenta transformation $v=\sqrt{x} y$. The energy relation (6) becomes now

$$
\text { (7) } \mathrm{v}^{2} / 2=1+\mathrm{hx} \text {, }
$$

and the time change is $d t / d T=x^{3 / 2}$ 
The phase portrait is very much like Fig. 1, except that now the orbits approaching collision have exactly 2 asymptotic finite values, since $\mathrm{v} \rightarrow \pm \sqrt{2}$ when $\mathrm{x} \rightarrow 0$. The collosion manifold $c$ consists of those 2 (equilibrium) points $(0, \pm \sqrt{2})$ glued to level curves for each fixed $h$. Topology of $E_{h}$ UC is a closed intervaI $[0,1]$ if $h<0$, and two half-open intervals $[0,1) \times S^{0}$ if $h \geq 0$.

For comparison with blow up, let us consider the regularization of binary collisions $x=0$ (Levi-Civita, Sundman). This is performed through the substitution $u=x y$, which gives a new energy relation.

$$
\text { (8) } u^{2} / 2=x+h x^{2}
$$

with a different change of time scale dt/d $\tau=x$ to take away the singularity in the transformed differential equations, getting a non homogeneous linear system

$$
\mathbf{x}^{\prime}=\mathrm{u}
$$

$$
\mathbf{u}^{\prime}=2 \mathrm{hx}+1
$$

Again the result is valid only for each fixed $h$. Now the orbits approaching collision go towards the origin in configuration space, which is not an equilibrium point, as we see from (9). Motion has been prolonged through $x=0$ like an elastic bouncing, contrasting blow up, where $x=0$ is approached in infinite $\tau$-time. The topology of a regularized $E_{h}$ is $s^{1}$ if $h<0$ (periodic motion) and $R$ if $h \geq 0$ (unbounded motion). 
In our 3- and 4-body examples to follow, binary collision singularities will remain after blow up of total collapse. A regularization can usually be applied, so that we assume it was performed.

\section{Example 2. Consider the isosceles (planar) three} body problem. We are given a particle of mass $\alpha$ moving along an axis and two particles of unit mass symetrically situated with respect to the axis. Using Jacobi-like coordinates the energy relation is

$$
\text { 0) } 1 / 2 \mathrm{pA}^{-1} \mathrm{p}^{t}=(2 \mathrm{x})^{-1}+2 \alpha\left(\mathrm{x}^{2}+\mathrm{y}^{2}\right)^{-1 / 2}+\mathrm{h} \text {, }
$$

where

$$
A=\operatorname{diag}(2,2 \alpha /(2+\alpha)) x>0, y \in \mathbb{R}
$$

This problem has been studied by several people, including Devaney, the author with Losco, and Simb.

The simplest triple collision orbits are the so-called homothetic solutions: nomotheties in time of the collinear or of the 2 possible equilateral triangular central configurations. Such orbits are regularizable, but without a continuous dependence on initial conditions to nearby orbits $|1|$.

From (4') we conclude that these 3 central configurations generate 6 -equilibrium points for the flow on $c$. Said points are hyperbolic for the flow on $E_{h} \cup C$ for any $\alpha$, all of them having invariant submanifolds of dimensions 1 and 
2. In Figure 2 we show the flow on $\mathrm{C}$ (which topologically is $S^{2}$ minus 4 points), the homothetic orbits and invariant submani folds near $C$. We assume $\alpha<55 / 4$ so that the flow on $c$ spirals at the equilibrium points on the symmetry axis. The gradientlike structure with respect to the "height" $v$ is also made clear.

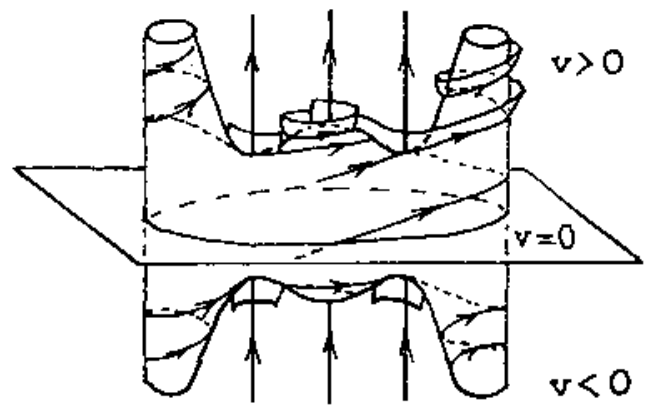

Fig. 2

As expected from our remark in the introduction : about motions for $h<0$, we can apply symbolic aynamics $|1|$ to show the existence of ejection - collision orbits, periodic orbits or orbits with some other kind of recurrence. We start by remarking that in this case respective homothetic orbits connect in $E_{h}$ (outside $C$ ) as shown in Figure 3 . The spiraling in variant manifolds intersect each other a countable number 
of times in the plane $v=0$. This implies the existence of heteroclinic orbits.
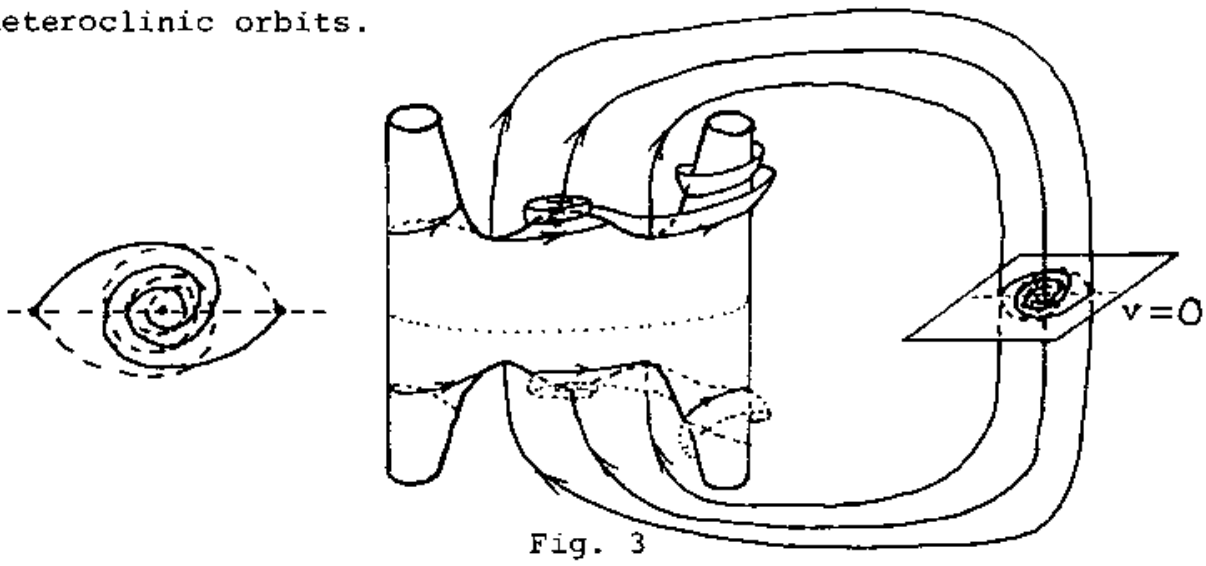

2.- Blow up at infinity $|7|$. - we have to consider here three cases, according to the sign of the energy.

If $\mathrm{h} \geq 0$ we modify the "polar coordinates" in section 1 by replacing $r$ by $\rho=r^{-1}$, so as to blow up the infinity of configuration space with an appropiate momentum transformation. The case $h<0$ is quite different, as we see below.

I) If $\mathrm{h}=0$, let $\mathrm{P}=\rho^{-1 / 2} \mathrm{p}$. This amounts to the substitution $r=\rho^{-1}$ everywhere in McGehee transformations, with energy relation

$$
\mathrm{K}(\mathrm{P})=\mathrm{U}(\mathrm{Q})
$$

and time change $d t=\rho^{-3} / 2 d \tau$, a speed up when $\rho \rightarrow 0$. The vector field is like (4), except that the first equation is replaced by $\rho^{\prime}=-\rho v$. It is also defined for $\rho=0$, winich 
defines an invariant infintty manifold $N_{0}$, where

$$
E_{0} \cup N_{0}=\{(\rho, Q, P): \rho \geq 0, Q \varepsilon S, X(P)=U(Q)\}
$$

In global terms we have an energy level with 2 boundary components: $E_{0} U N_{0} U C$. In this case the flow is projectable on $\mathrm{C}$ or $\mathrm{N}_{0}$ along $r$ or $\rho$, in a sense which can be made precise.

We can check that the flow on $N_{0}$ is identical with that on $C$ and equilibrium points are the same. This recovers the classical result that for zero energy, escape to infinity is asymptotically identical to total collapse.

II) If $\mathrm{h}>0$ the above transformation does not work, since energy relation (3) with $r=\rho^{-1}$ is singular for $p=0$. We change momenta transformation to the trivial one $\mathrm{p}=\mathrm{p}$, which gives an already regular energy relation

$$
\text { (1I) } K(P)=\rho U(Q)+h \text {, }
$$

nontrivial at $\rho=0$ only if $h>0$.

In the new coordinates $\rho, 0, P$, differential equations annihilate for $\rho=0$. We speed up orbits with the change of time scale dt $=\rho^{-1} d \tau$ to get the following system of equations

$$
\text { (12) } \begin{aligned}
\rho^{\prime} & =-\rho \mathrm{v} \\
\mathrm{Q}^{\prime} & =\mathrm{PA}^{-1}-\mathrm{v} \\
\mathrm{P}^{\prime} & =\rho \text { grad } \mathrm{U}(Q)
\end{aligned}
$$


The flow is again defined and nontrivial for $p=0$, having a corresponding infinity manifold $\mathrm{N}_{\mathrm{h}}$, where

$$
\begin{aligned}
& E_{h} \cup N_{h}=\{(\rho, Q, P): \rho \geq 0, Q \varepsilon S, K(P)=\rho U(Q)+h\} \\
& \text { In this case the equilibrium points for } \rho=0 \text { on } N_{h}
\end{aligned}
$$

form two submanifolds

$$
\mathrm{S}_{\mathrm{h}} \pm=\{(\rho, Q, P): \rho=0, Q \in \mathrm{S}, \mathrm{P}= \pm \sqrt{2 \mathrm{~h}} \mathrm{QA}\} \subset \mathrm{N}_{\mathrm{h}^{\prime}}
$$

with a very simple flow structure studied by the author $|3|$ :

Proposition 2.- The vector field on $E_{h} U N_{h}$ has $s_{h}^{+}$ as normally hyperbolic submanifolds. In fact, $S_{h}^{+}$is an attractor and $s_{h}^{-}$is a repellor. Besides, the flow on $\mathrm{N}_{h}$ is gradient-like with respect to $v$.

Any asymptotic direction of escape to infinity is possible, since $s_{h}^{+}$(and $s_{h}^{-}$) has one point for each direction $Q \varepsilon S$. This is in contrast with the case $h=0$, where only central configurations are possible. That the flow is gradient like with respect to $v$ is seen by checking that $v^{\prime}=Q^{\prime} A Q^{\prime} \geq 0$ if $\rho=0$, with $v^{\prime}=0$ exactly at equilibrium points.

We consider again the examples in $\$ 1$.

Example 1. - Set $z=x^{-1}$ in the energy relation (6) 
for the Kepler problem. We get

$$
\text { (13) } y^{2} / 2=z+h \text {, }
$$

with the change of time scale $d t / a t=z^{-1}$. This is a transfor mation type II, good for $h>0$.

From (13), we see that solutions are parabolas $|4|$, truncated by equilibrium points at $x=0$ (since $h>0$ ), because of the condition $x \geq 0$. We can now give the complete topology of $E_{h}$ UCUN $_{h}$, as two closed intervals $[0,1] \times s^{\circ}$, the endpoints being of course equilibria.

The description in the new coordinates is still good for $h<0$, only that the motion is then bounded (relation (13) gives parabolas bounded away from $z=0$ ), and there is no infinity manifold.

Example 2. The topology of $E_{h} U_{h}$ UC for $h \geq 0$ in the planar isosceles 3 -body problem is $\left(S^{2}-4\right.$ points) $x[0,1]$. However, the flow on $\mathrm{E}_{\mathrm{h}} \mathrm{UN}_{\mathrm{h}}$ changes with $\mathrm{h}$. If $\mathrm{h}=0, \mathrm{~N}_{\mathrm{h}}$ has 6 critical points as in Fig. 2; for $h>0$ we have two curves $\mathrm{s}_{\overline{\mathrm{h}}}^{+}$of critical points.

Consider the case $h=0$. Because of projectability of the flow, the invariant submanifolds of hyperbolic equilibrium points on $\mathrm{N}_{\mathrm{O}}$ and $\mathrm{C}$ connect each other across $\mathrm{E}_{\mathrm{O}}$. In Fig. 4 a) we show one of those submanifolds $w$, which comes from $c$, while 
its outer orbit $\Gamma$ is in $N_{o}$. There is an orbit $\gamma$ in $w$ coming from collinear configuration escape as $t \rightarrow-\infty$ (with oscillations if $\alpha\langle 55 / 4\rangle$, asymptotically ending up in an equilateral configu ration escape as $t \rightarrow+\infty$, passing as close as we like to triple collision. The projection of $\gamma$ to configuration space is shown in Fig. 4b).

Similar motions in the collinear 3-body problem for $h=0$ were considered in $|7|$.

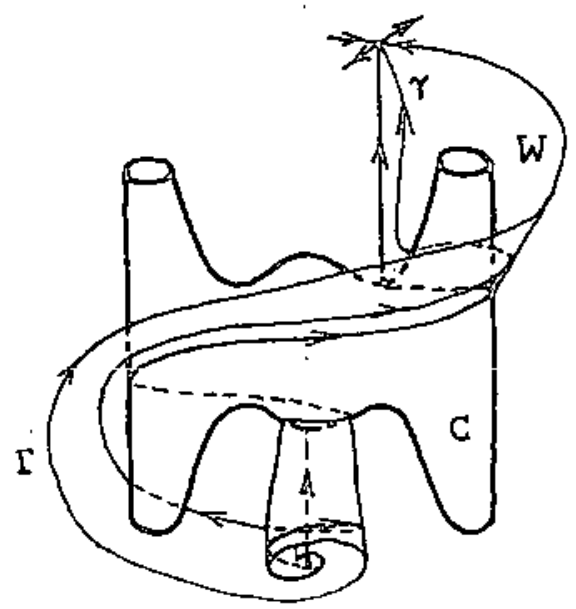

a)
Fig. 4

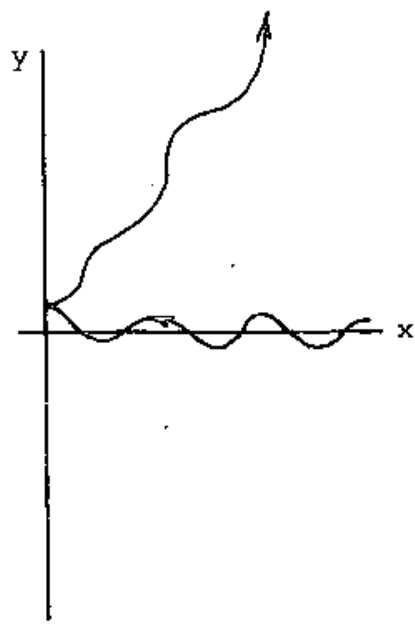

b)

III) If $\mathrm{h}<0$ the situation is more complex, since at least one of the mutual distances $r_{i j}$ must be bounded, because energy relation (2) implies $\mathrm{U} \geq-\mathrm{h}>0$. Hence, the above spherical blow ups along rays $Q \in S$ do not work here, since for one thing they do not distinguish at infinity between bounded motions and collisions. 
Another way to see that is by observing that $u(q)+0$ when $q \rightarrow \infty$ on a fixed ray, because of homogeneity of $U$. If in addition $i<0$, eventually there is no value of p satisfying (2) and hence, no motion (see |6| for a detailed discussion).

In the particular case $n=2$ bodies there is no infinity manifold, because $\left.\mathrm{U}\right|_{\mathrm{s}}$ is bounded and all the negative energy solutions are bounded.

The answer for $n \geq 3$ bodies in general is to keep track of all the possible partitions into particle clusters, to construct the different patches of the infinity manifold.

We will sketch the ideas of the modified blow ups for the isosceles $|7|$ and the trapezoidal $|3|$ problems on negative energy levels, but our method is valid for $n \geq 3$ boay problems in the plane or the space (see the forthcoming paper $|5|)$.

Example 2.- Coming back to the isosceles problem, we can see from energy relation (10) that if $h<0$ escape occurs only for $y \rightarrow+\infty$ or $y+-\infty$.

We let $Y=\rho^{-I}>0$ for the first possibility, which can be interpreted as blowing up of a portion of the $x$-axis, transforming (10) into the form

(14) $1 / 2 \mathrm{pA}^{-1} \mathrm{p}^{t}=(2 \mathrm{x})^{-1}+2 \alpha \rho\left(1+\rho^{2} \mathrm{x}^{2}\right)^{-1 / 2}+\mathrm{h}$. 
We see that no momenta transformation is needed, since (14) is regular for $\rho=0$. No change of tine scale either, as we check from the transformed differential equations. The flow at the corresponding infinity manifold $\mathrm{N}_{h}^{+}$describes an unatching at infinity of a binary with fixed negative energy, going away with constant velocity from the third particle.

We have similarly a symetrical component $\mathrm{N}_{\mathrm{h}}$ of infinity manifold by letting $y=-\rho^{-1}<0$. The topology of $E_{h} U N_{h}^{+} \cup N_{h}^{-} U C$ in this case is more complicated than before: $E_{h}$ is a 2-hole solid torus, $\mathrm{N}_{\mathrm{h}}^{+}$and $\mathrm{N}_{\mathrm{h}}^{-}$are both topologically $S^{2}-2$ points, and $C=S^{2}-4$ points as before. See $|4|$ for the descriptive figure.

\section{Example 3.- Consider now the trapezoidal} 4-body problem in the plane. In this case we are given 4 particles: two of unit mass symmetrically situated with respect to an axis in the plane. The other 2 particles of mass $\alpha$ are also symetrically located with respect to the axis. Using Jacobi-Iike coordinates $x, y>0$ and $z \varepsilon I R$, the energy relation reads as

$$
\mathrm{p}_{1}^{2} / 4+\mathrm{p}_{2}^{2} /(4 \alpha)+\left(1+\alpha^{-1}\right) \mathrm{p}_{3}^{2} / 4=(2 \mathrm{x})^{-1}+\alpha^{2} /(2 \mathrm{y})+2 \alpha / \mathrm{R}_{1}+2 \alpha / \mathrm{R}_{2}+\mathrm{h}
$$

where $R_{1}=\sqrt{(x-y)^{2}+\bar{z}^{2}}, \quad R_{2}=\sqrt{(x+y)^{2}+z^{2}}$. 
A description of the quadruple collision manifold $C$ in this problem has been made by the author.

To get an idea of the behaviour at infinity for $\mathrm{h}<0$, we see that the Hill region in configuration space $\left(\mathbb{R}^{+}\right)^{2} \mathbf{x} \mathbb{R}$ is limited by the half-planes $x=0, y=0$ and the zero velocity surface $\mathrm{U}=-\mathrm{h}$ which is asymptotic to the cylinder $\mathrm{R}_{1}=-2 \alpha, \mathrm{h}$ and to the simpler surface $(2 \mathrm{x})^{-1}+\alpha^{2} /(2 \mathrm{y})=-\mathrm{h}$ (two components). The latter is in turn asymptotic to both half-planes $x=-(2 h)^{-1}$ and $y=-\alpha^{2} /(2 h)$.

For $|\mathrm{h}|$ big enough, we can change the notion of rays by using the $\mathrm{C}^{\infty}$ smoothing out at corners of the new sphere shown in Fig. 5, instead of the unit "sphere" $s$ in (5). Intersection with the Hill region are the 2 shaded pieces, giving the components of the infinity manifold.

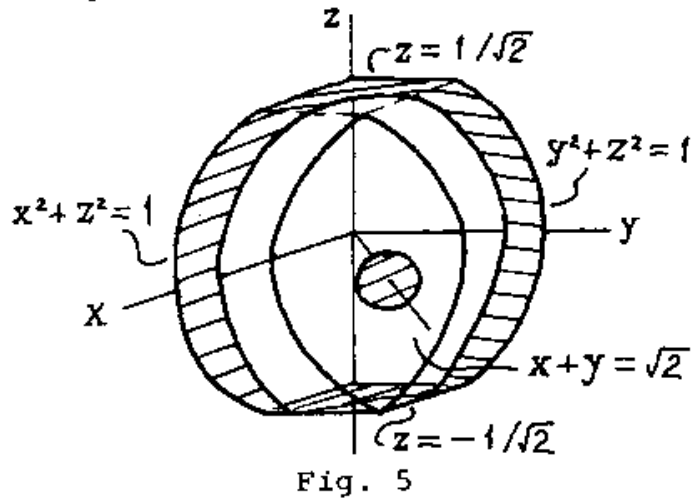

This unit sphere defines a new norm or radial coordinate in configuration space, which amounts in each patch to a blow up of a portion of the $x y$ plane through $\pm^{-1 / 2} z^{-1}$; of a portion of the $x$-axis through $\left(y^{2}+z^{2}\right)^{-1 / 2}$, etc. Computing 
the flow in each of these 2 cases $|5|$, we get an unmatching into 2 kepler (regularized) collinear problems in the ficst case. In the second case particles of mass $\alpha$ escape eacin one on its side like 2 individual clusters whose center of mass moves with uniform velocity away from the negative energy binary cluster of unit mass particles.

The 2 components of infinity manifold are topologically $\mathrm{N}_{\mathrm{h}}^{1}=\mathrm{S}^{1} \times \mathrm{S}^{1} \times \mathrm{D}^{2}-\mathrm{D}^{2} \times \mathrm{S}^{0}$ and $\mathrm{N}_{\mathrm{h}}^{2}=\mathrm{S}^{4}-2$ points, where $\mathrm{D}^{2}$ is the open 2-ball.

3. Extension to other force laws.- The above blow up procedures can be extended to mechanical systems (1) where U is a homogeneous function of any degree $k \in \operatorname{IR}$ (see |6!).

The simplest examples keeping $k=-1$ as in celestial mechanics are repulsive n-body problems (with positive charged particles), or with a combination of attractive and repulsive coulombian forces (different sign charged particles). We get the following variations of the isosceles plane (gravitationa1) 3 body problem of Example 1 .

Example 4.- The isosceles plane repulsive 3-body problem, where we just replace the function $U$ in (10) by $-U$, getting the energy relation

$$
\text { (14) } 1 / 2 \mathrm{pA}^{-1} \mathrm{p}^{t}=-(2 \mathrm{x})^{-1}-2 \alpha\left(\mathrm{x}^{2}+\mathrm{y}^{2}\right)^{-1 / 2}+\mathrm{h}
$$


with A as before. This corresponds to a charged particle of charge $\alpha>0$ moving along an axis and two particles of unit charge symmetrically situated about the axis. In this case we always have $\mathrm{h}>0$ with no collisions, and typical Hill regions in configuration space are shown in Fig. 6. Case a) corresponds to the limiting case $\alpha=0$ (restricted problem), which has been studied by Peredo $|10|$.

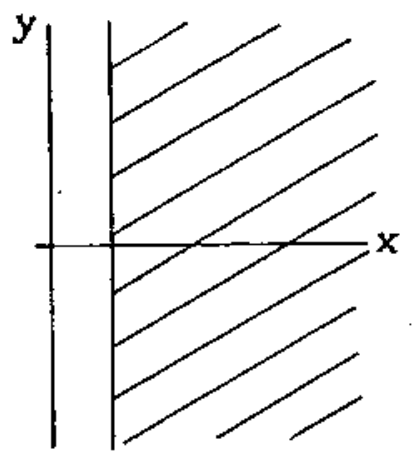

a) $\alpha=0$

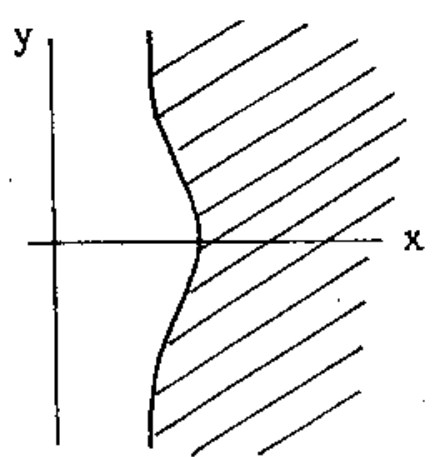

b) $\alpha>0$

Fig. 6

Example 5. - The plane isosceles Helium atom problem $|6|$, where symmetrical particles in example 4 have now charge -1. The energy relation becomes

(15) $1 / 2 \mathrm{pA}^{-1} \mathrm{p}^{t}=-(2 \mathrm{x})^{-1}+2 \alpha\left(\mathrm{x}^{2}+\mathrm{y}^{2}\right)^{-1 / 2}+h$

There are 3 different cases as shown in Fig. 7, where some typical Hill regions are shaded.Collisions do occur only 
in case a) for $h<0$, as in the case of the shaded shown sector. The reason is that the positive charge is big enough to overcome the repulsion of the others

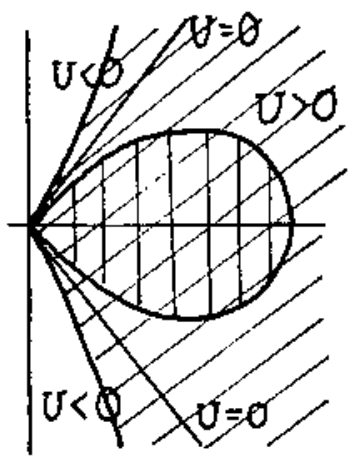

a) $\alpha>1 / 4$, any $h$

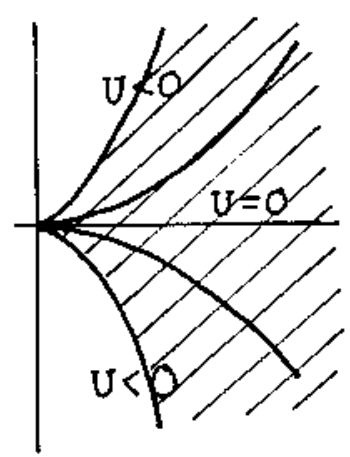

b) $\alpha=1 / 4, \mathrm{~h}>0$

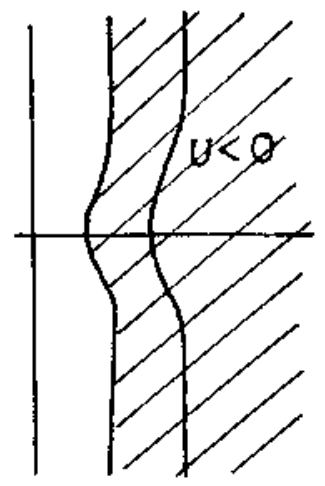

c) $\alpha<1 / 4, \mathrm{~h}>0$

Fig. 7

REFERENCES

1. R. Devaney, Singularities in Classical Mechanical Systems, in Ergodic Theory and Dynamical systems I, Birkhauser Boston 1981, 211-233.

2. R. Devaney, Blowing up singularities in classical mechanical systens, Amer. Math. Monthly, 89 (1982) 535-552.

3. E. Lacomba, Varietes l'infini pour une Energie non nulle en mécanique céleste, C.R.A.S. Paris I 295 (1982) 503-506.

4. E. Lacomba, Infinity manifolds on energy levels for celestial mechanics, in proc. Nonlinear

Phenomena, K.B. Wolf ed. Springer Verlag, 1983.

5. E. Lacomba, Negative energy infinity manifolds in 3 and 4-body problems, to appear 1984.

6. E. Lacomba, Origin and infinity manifolds for mecha nical systems with homogeneous potentials, to -F appear 1984 . 
7. E. Lacomba and C. Simó, Boundary manifolds for energy surfaces in celestial mechanics, Celestial Mechanics 28 (1982) 37-48.

8. T. Levi-Civita, Sur la résolution qualitative du problème restraint des 3 corps, Opere Mathematiche 2 (1906).

9. R. McGehee, Triple collision in the collinear three body problem, Invent. Math. 27(1974)191-227

10. F. Peredo, El problema restringido isósceles de 3 cuerpos con fuerzas repulsivas, to appear, 1984.

Rebut el 16 de maig del 1984

Departamento de Matemáticas

U.A.M. , Iztapalapa

Apdo. Postal 55-534

09340 México, D.F. 